\title{
Entendimiento conceptual de los estudiantes del nivel de básica secundaria sobre el concepto de ácido*
}

\author{
Roberto Figueroa Molina \\ Carlos Utria Echeverría \\ Rafael Colpas Castillo**
}

Artículo recibido: 11-05-2005 y aprobado: 26-04-2006

Resumen: Los resultados que se presentan en este estudio se encuentran enmarcados en el paradigma constructivista; tuvo como finalidad indagar el entendimiento conceptual de los estudiantes de educación básica y media sobre la química de los ácidos. La investigación realizada se describe como fenomenológica, debido a que atiende no sólo a las características de las concepciones sino también su fuente de origen, basadas en las creencias, intenciones y motivaciones de quienes participaron en el proceso educativo. Es decir, con este diseño se apunta a trabajar en función de las concepciones que poseen los estudiantes sobre los ácidos mediante entrevistas apoyadas en actividades, como la clasificación de tarjetas y la solución de situaciones problemáticas, para así lograr en ellos cambios a nivel conceptual, procedimental y actitudinal.

Palabras clave: Concepto de ácido, entendimiento conceptual, estudiantes de escuela secundaria, clasificación de tarjetas y solución de situaciones problema.

Secondary school students' conceptual
understanding regarding the acid concept

Abstract: This study was aimed at inquirying about secondary students' conceptual understanding regarding the acid concept and its results were framed in a constructivist paradigm.

The research is considered a phenomenological study, due to the fact that its focus is not only related to the characteristics of conceptions, but also to their origin source, having in mind the intentions, believes and purposes of those who participated in the educational process. Whit this design it is expected to work on the conceptions students have about acids, by using interviews that include activities such as cards classification and problem solving to achive a conceptual and attitudinal change.

Key words: Acid concept, conceptual understanding, secondary students, card classification and problem solving.

\footnotetext{
Trabajo presentado en el I Coloquio Nacional de Enseñanza de las Ciencias. Ibagué, 2004.

** Grupo "GECIT", Universidad del Atlántico. Roberto Figueroa Molina, rfiguero@metrotel.net.co Carlos Utria Echeverría,c_utria@hotmail.com,Rafael Colpas Castillo,rafacolpas@latinmail.com
} 


\section{Introducción}

Un aspecto importante de la educación que en los últimos tiempos ha sido motivo de reflexión de muchos educadores es la tendencia de los estudiantes a explicar los fenómenos que suceden a su alrededor utilizando sus ideas y no las científicas impartidas en la enseñanza formal. Tales ideas son denominadas concepciones alternativas, que los estudiantes construyen para interpretar y explicar sucesos naturales cotidianos.

Estas concepciones han sido motivo de investigación desde finales de la década de los sesenta debido a que, por un lado, son distintas a las explicaciones científicas y, por el otro, no son ideas simples que cambien al enseñar ciencia en la escuela. Su persistencia puede durar muchos años, e incluso hasta el nivel universitario, en el cual se han encontrado estudiantes que presentan creencias no correspondientes con lo que se les ha enseñado en sus cursos de Ciencias Naturales (Fensham, et al., 1994).

Las investigaciones sobre comprensión de conceptos en ciencias experimentales condujeron muy rápidamente a distintos autores a verificar la hipótesis más plausible de la existencia en los niños de ideas sobre temas científicos previas al aprendizaje escolar y que fueron designadas como ideas ingenuas (Taramazza, 1981), ciencias de los niños (Gilbert, 1982; Osborne y Wittrock, 1983), esquemas conceptuales alternativos (Driver y Easley, 1978), representaciones (Giordan, 1985)' ${ }^{1}$, etc.

Por los resultados obtenidos de investigaciones en didáctica de las ciencias experimentales (Carr, 1984;

1 www.campus-oei.orgioelvirt/gil02.htm
Hewson, 1984; Giordan y De Vecchi, 1988, y Pozo et al., 1991) sobre las dificultades que frecuentemente presentan los estudiantes de diferentes niveles académicos en su enseñanza habitual, se ha podido percibir que éstas se encuentran relacionadas con las estrategias empleadas por el docente en el desarrollo de las temáticas en esta área. Todas estas percepciones pueden ser causadas por la falta de conocimiento de los profesores respecto a las concepciones que poseen los estudiantes de los temas estudiados.

Según Barrios (1997), la falta de atención de los docentes hacia lo que el estudiante puedan pensar, y los obstáculos que dichas ideas puedan representar para la enseñanza, traen consigo los resultados tan negativos de la misma. Lo anterior proporciona evidencias que demuestran que la mayoría de los estudiantes se han preparado con un modelo educativo de corte informativomemorístico.

Para el caso de la temática ácidos, tales apreciaciones están sustentadas por los estudios realizados por Carr (1984) y Hand (1989), quienes encontraron que los estudiantes, después de haber recibido la enseñanza sobre las características de los ácidos, aún tenían ideas preconcebidas sobre lo que es un ácido y cómo se reconoce si algo es un ácido.

Fensham (1994) y Hand y Treagust (1989) coinciden en sus estudios que las concepciones más relevantes sobre ácidos fueron los "ácidos corroen el material" y "los ácidos pueden quemarte". Comprobaciones posteriores revelaron otras dos ideas ampliamente mantenidas de que "la única prueba respecto a 
un ácido es ver si corroe algo" y que "los ácidos fuertes corroen el material más de prisa que los ácidos débiles". Asimismo, se encontró que los estudiantes no perciben las reacciones de los ácidos con los metales o con el carbonato de calcio como propiedades de un ácido. En su lugar, las consideraban como ejemplos adicionales de "ácidos corroyendo algo". $\mathrm{Al}$ examinar a estos estudiantes unos dos años después de que habían recibido la enseñanza sobre las características de los ácidos, se encontró que puntuaban peor la segunda vez en las tareas relacionadas con definir el término ácido y comprobar si algo es un ácido; sin embargo, puntuaban mejor al definir la diferencia entre fuerza del ácido y concentración del ácido. Al respecto Borsese (1992) destaca la importancia de utilizar correctamente el lenguaje y el significado de las palabras para ser rigurosos y precisos cuando nos referimos a los conceptos de química en el caso concreto de los ácidos y la fuerza de éstos. Todos estos hechos se presentan en gran parte porque los docentes no realizan actividades efectivas para identificar las concepciones alternativas que poseen los estudiantes sobre ácidos. Por tanto, éstas no son tomadas en cuenta, con el consiguiente resultado de que persistan aún después de la enseñanza.

En otras palabras, las estrategias utilizadas por los docentes no son suficientemente motivantes, y esto causa desinterés en los estudiantes teniendo en cuenta que un alto porcentaje de ellos encuentran que la idea original de ciencias que traen a clases no concuerda con lo impartido en el aula. Es decir, de experiencias directas (concepciones) obtenidas de ámbitos no formales, pasan al trabajo en el aula o en el laboratorio, donde reciben contenidos aplicados con rigurosidad científica. Ello dificulta la comprensión de los mismos fenómenos que antes se presentaban bajo formas creativas y originales ${ }^{2}$.

La nueva forma de enseñar debe proponerse, por tanto, que los alumnos modifiquen sus ideas. Tal pretensión no se considera exenta de dificultades, pues se trata de un proceso complicado en el cual las tensiones entre lo existente y lo nuevo desempeñan un papel fundamental, similar al que se produce en los cambios de paradigmas científicos. El nuevo enfoque metodológico insiste en la necesidad de que los profesores estén al tanto de las ideas previas de los alumnos y empleen estrategias que favorezcan la creación de conflictos cognitivos entre las ideas espontáneas y las ideas científicas, a fin de lograr el deseado cambio conceptual.

En consecuencia, el presente estudio surge de las interacciones con los estudiantes de colegios oficiales de bachillerato de centros de educación básica y media de Barranquilla, en las cuales se pudo apreciar, a través de las experiencias realizadas, que los estudiantes presentan dificultades para reconocer las características que permiten clasificar una sustancia como ácido, las relaciones con otros conceptos químicos, así como también las confusiones que presentan al intentar prever las posibles reacciones que pueden darse al mezclar sustancias ácidas y básicas, o ácidos con otras sustancias como los carbonatos.

2 www.unesco.cl/pdf/actyeven/ppe7boletin/ artesp/44-4.pdf 
Los planteamientos aludidos evidencian que una de las metas de los profesores debe ser una enseñanza que provoque o promueva la comprensión de conceptos (Platten, 1991). Lo mismo sostiene Nakhleh (1994), acerca de cómo los estudiantes aprenden química. Este autor sugiere que se deben utilizar modelos instruccionales adecuados para ayudar al estudiante a integrar sus observaciones con su entendimiento conceptual.

\section{Método}

El estudio utilizó un diseño descriptivo basado en un enfoque fenomenológico de interpretación cualitativa. El propósito del estudio fue determinar el grado de comprensión de los estudiantes sobre las características química de los ácidos, mediante entrevistas a través de actividades, como la clasificación de tarjetas y solución de situaciones problemáticas. La muestra estudio estuvo conformada por estudiantes de educación básica y media de colegios oficiales del Distrito de Barranquilla, atendiendo al nivel académico excelente $(\mathrm{n}=8)$, aceptable $(\mathrm{n}=8)$ e insuficiente $(\mathrm{n}=8)$, de cada uno de ellos en la asignatura de química. Esto debido a que el uso de muestras pequeñas es adecuado al propósito de este estudio cualitativo que va dirigido a incrementar el entendimiento de los fenómenos y no a generalizar los resultados (Maykut y Morehouse, 1994).

\section{Recopilación de datos}

Por las características cualitativas de esta investigación, se empleó una entrevista con profundidad de carácter individual; para cada entrevista se manejó un protocolo con preguntas tales como: "Ante una sustancia dada, ¿qué caracte- rísticas tendría en cuenta para establecer que se trata de un ácido? Según los criterios establecidos en las actividades desarrolladas, ¿cómo definiría un ácido?". La entrevista se realizó teniendo en cuenta las actividades preliminares, como la clasificación de tarjetas y la solución de situaciones problema. Para la primera actividad se utilizaron 18 tarjetas ilustradas con una serie de sustancias, que cada estudiante debía clasificar atendiendo al criterio que poseían sobre los ácidos. En la segunda parte de esta actividad, a los estudiantes se les proporcionó una gráfica de barras de $\mathrm{pH}$, en la cual ellos debían ubicar las sustancias ácidas que clasificaron en la primera parte de la actividad, atendiendo a los criterios que tenían sobre el pH de dichas sustancias.

En la solución de situaciones problema se incluyeron dos asignaciones y una práctica de laboratorio. En la primera asignación se plantearon cinco situaciones problema, que buscaban propiciar en cada estudiante un conflicto cognitivo que lo llevara a inferir qué fenómeno surgiría en la situación planteada. Tal actividad estuvo conformada por problemas como: "Si colocas un huevo dentro de un recipiente que contiene vinagre, ¿qué sucederá?". Una vez realizada la primera asignación, se llevó a cabo una experiencia de laboratorio que mostraba los fenómenos que ocurrían en las situaciones problema presentadas. Culminada esta experiencia se dio paso a la segunda asignación en la que se entrevistaron a los estudiantes, se les plantearon las mismas situaciones, pero con la variante de que ellos debían ofrecer una explicación para la ocurrencia del fenómeno observado, 
con la finalidad de indagar sobre las concepciones de cada estudiante sobre las reacciones de los ácidos.

\section{Análisis de datos}

El análisis cualitativo se desarrolló a partir de las concepciones de los estudiantes, obtenidas de los protocolos de las entrevistas y de la solución de situaciones problema sobre la química de los ácidos, las cuales se ordenaron en categorías de análisis, apoyadas en los estudios de Pozo, Sanz, Gómez y Limón (1991), quienes consideran que las concepciones alternativas pueden ser espontáneas, inducidas y analógicas. El grupo de las espontáneas incluye aquellas que son producto de la percepción de experiencias y observaciones de la vida cotidiana. Las inducidas son el resultado de las interacciones de los estudiantes con su entorno social y cultural donde se pueden señalar como fuentes de primera mano la familia, la escuela y los medios de comunicación. Las concepciones analógicas se presentan cuando los estudiantes recurren a ideas ya existentes para explicar un fenómeno nuevo y que muchas veces no guardan relación entre sí, sino que son creadas por el mismo estudiante o como producto de la enseñanza.

\section{Concepciones espontáneas}

Los estudiantes manifiestan concepciones que pueden identificarse como espontáneas, puesto que se basan en experiencias personales de tipo sensorial y observacional. Ellos fundamentan sus concepciones en "lo que se ve es lo que se cree", y por tanto estas concepciones suelen ser incorrectas y ajustadas a determinadas situaciones independientes y no relacionadas con otras situaciones de un mismo tema o concepto. Los estudiantes, para definir el concepto de ácidos e identificar sustancias de este tipo, se basan en aspectos sensoriales como el olor, sabor y color de las sustancias. Tal como lo sustenta Carr (1984), quien afirma que las concepciones de los estudiantes acerca de los ácidos derivan de experiencias sensoriales, como probar leche cortada, limones o vinagre, Italo en su entrevista expresa: "Un ácido puede distinguirse por el sabor, como el limón cuando uno lo prueba siente algo feo... También por los colores, sé que es un ácido; para mí el color verde es un ácido casi siempre... además a mí un mal olor me hace pensar que eso es ácido, pero no un ácido natural sino por bacterias acumuladas".

Por su parte, Alba menciona que: "Los ácidos son sustancias que se reconocen por su sabor... fuerte, ya sean líquidos o gaseosos; los productos que son polvos, como la ceniza no los considero ácidos".

Las concepciones alternativas expuestas son diferentes a los conceptos científicos en el sentido de que no establecen definiciones sino enunciados descriptivos o explicativos; por tanto estas concepciones alternativas se deben entender como simples enunciados que hacen alusión a la interpretación de un proceso que no tiene en cuenta lo científico ${ }^{3}$.

Otro rasgo característico de la comprensión de la química de los ácidos, por parte de los estudiantes al inte-

3 http://ideasprevias.cinstrum.unam.mx:2048/base. htm 
rrogarlos sobre las reacciones típicas de los ácidos. Se destaca la presencia en ellas de una continuidad espaciotemporal entre causa y efecto, producto del predominio de la percepción en la formación de las concepciones alternativas sobre las reacciones de los ácidos. Podemos citar lo expresado por Ana, quien explica: “Al introducir un huevo en vinagre esto empieza a efervescer, luego sube poco a poco el huevo y se va poniendo blanco, porque el ácido que contiene el vinagre hace que la cáscara afloje el color que posee el huevo... o sea se desolleja”. Del mismo modo Emma, aunque haciendo mención a otro fenómeno afirma: “Al agregar el ácido del vinagre a la leche de magnesia y a la vez un indicador natural, la leche se corta debido al ácido del vinagre y toma un color como verdoso".

Estas interpretaciones no coinciden con la visión científica sobre las reacciones de los ácidos y otras sustancias, como las reacciones entre carbonatos y ácidos y las de neutralización. Es decir, no tienen en cuenta que pueden ocurrir reacciones intermedias no observables que generan efectos que sí se pueden percibir. Se observa que su entendimiento conceptual se fundamenta meramente en el aspecto observacional. Los estudiantes explican los fenómenos que observan como ejemplos de un ácido "corroyendo" algo, mas no como la interacción entre dos sustancias diferentes. Esto evidencia que los estudiantes sólo especifican la acción que puede causar una sustancia ácida, pero no las reacciones que se pueden presentar entre éstas con otras sustancias, como en el caso de las bases. Solamente afirman: "Los ácidos no reaccionan con las bases; sólo se mezclan" ${ }^{4}$. Igual puede apreciarse en el relato de Isaac, quien considera que: "Creo que al echar el zumo de limón a una superficie de mármol, poco a poco el ácido del limón se va comiendo el mármol".

\section{Concepciones inducidas}

Los medios de comunicación, la interacción con amigos y familiares e incluso con los profesores constituyen una fuente de información de primera mano para los estudiantes. De allí obtienen ideas que fundamentan sus creencias y con las cuales forman sus conceptos sobre los fenómenos que observan. Vygotsky (1982) plantea que el crecimiento intelectual de los estudiantes depende del dominio de los medios sociales, esto es, del lenguaje. Al respecto Enrique, declara: “A mí me dijeron una vez que si alguien está pintando con cal y le cae en la vista... se le daña la retina; entonces por eso pienso que la cal es un ácido, si dicen que no puede caer en los ojos y por eso los que pintan se ponen gafas, para evitar la acidez de la cal”.

La idea anterior refleja la influencia del lenguaje cotidiano y cómo a través de éste cobran fuerzas las creencias personales que terminan interfiriendo con el lenguaje científico, ya que para los estudiantes prima el saber popular porque en él encuentran explicaciones acordes con su realidad (Cazden, 1991). Es decir, que las concepciones socialmente inducidas más estudiadas son las que se deben al efecto del lenguaje y a las formadas por medio de lo que la

4 http://ideasprevias.cinstrum.unam.mx:2048/base. htm 
psicología social denomina representaciones sociales (Solomon, 1987).

Podemos inferir entonces que el pensamiento alternativo surge cuando los estudiantes interiorizan las experiencias que viven, en las cuales lo importante es que las ideas se ajusten a una situación determinada independiente de la interrelación que ésta pueda tener con otras situaciones. Driver (1988) plantea: "Lo que se aprende no depende solamente de las características del conocimiento presentado por los medios de comunicación y los libros de texto, sino de los esquemas que tiene disponible el que aprende". En otras ocasiones los medios de comunicación influyen sobre la formación de determinados conceptos, puesto que se considera que el mundo de la publicidad puede actuar como una fuente social para las concepciones con las que los estudiantes acceden a las aulas. Todo esto debido a que el elevado consumo de propaganda que realizan los jóvenes por la televisión y otros medios de comunicación -basado en la imagen y la estética publicitaria- convierten estos medios en un agente informal de educación de gran relevancia (Trilla, 1993).

Eleucilio, señala que: "El jabón es un ácido porque mata gérmenes, lo dice en la etiqueta... y en la televisión también lo muestran". Hand (1988), afirma que los estudiantes ajustan sus ideas a situaciones muy específicas, ofreciendo explicaciones que a su modo de ver resultan ser claras, coherentes y convincentes, tales como "Los ácidos fuertes corroen el material".

\section{Concepciones analógicas}

En lo referente a la química de los ácidos, muchas de las concepciones de los estudiantes proceden de inter- pretaciones propias o de analogías que para ellos son coherentes y encajan con ciertas situaciones "reales" o abstractas. Muchas de estas analogías son creadas. Como afirma Juan: "La crema dental es un ácido porque es capaz de matar gérmenes y con el contacto con la piel... quema. En tanto el limpiador de cañerías, el ácido que contiene mata gérmenes debido a que está hecho para destapar tuberías".

Lo anterior muestra cómo los ácidos corroen los materiales y cómo esta capacidad les permite ser aptos para matar los gérmenes que se encuentran en ciertos lugares. Hand y Treagust (1988), encontraron que las dos concepciones más importantes de los estudiantes sobre los ácidos son "Los ácidos corroen el material" y "Los ácidos pueden quemarte".

Del mismo modo, los estudiantes convierten sus concepciones en verdades indiscutibles porque en ellas encuentran seguridad, como se aprecia en lo que plantea Enrique: "Yo pienso que el champú no es un ácido, porque si el champú fuera un ácido, nadie en este mundo tendría cabello... se le dañaría el cuero cabelludo". Otra característica del entendimiento de la química de los ácidos es la tendencia a razonar a partir de las características observables de las sustancias, al manifestar la existencia de sustancias ácidas naturales que se reconocen por el sabor o por su forma: "El tomate no es un ácido porque es dulce y tiene la concha blandita; por eso creo que no es... mientras que el limón tiene la concha dura y su sabor es agrio; por eso sé que es un ácido".

La analogía basada en experiencias sirve de puente para diferenciar los ácidos fuertes y los débiles. "El vinagre es 
más ácido que el limón porque tiene un olor más fuerte al igual que su sabor". También fundamentan interpretaciones equivocadas para identificar sustancias ácidas “....un efecto de los ácidos es que irrita los ojos; el limpiador de cañerías, por ejemplo, tiene la capacidad de irritar los ojos... además es fuerte, derrite las cosas, las daña". "Los antiácidos son cosas, sustancias que no deben contener ácidos, no se deben mezclar entre ellos". Igualmente, manifiestan que los ácidos pueden desmanchar, despercudir o quitar sustancias que hay en ciertos lugares, pero que de igual manera causan daño en muchas ocasiones.

Estas concepciones, según Carr (1984), proceden en muchos casos de las historias de crímenes sobre los baños ácidos, anuncios de remedios antiácidos y noticias sobre los efectos de la lluvia ácida.

\section{Conclusiones}

El análisis de las evidencias encontradas en el estudio nos llevan a concluir que los procesos de enseñanza y aprendizaje deben promover la construcción de estrategias didácticas que permitan identificar el entendimiento de los estudiantes acerca de cualquier concepto, proposición, tema o idea, antes de iniciar la enseñanza, con el objeto de utilizarlas como punto de partida en la asimilación de los conceptos científicos (Ausubel et al., 1990), para que los estudiantes puedan transformar las concepciones en ideas más adecuadas y congruentes con el conocimiento científico (Hewson, 1984). Las concepciones alternativas de los estudiantes no cambian, sino evolucionan o se amplían a lo largo de la escolarización hasta llegar a ser coherentes con la ciencia escolar. Es decir, todo lo que el estudiante aprende es "con" y "contra" sus propias concepciones (Giordan y De Vecchi, 1988).

La entrevista apoyada en actividades permitió indagar eficientemente sobre el entendimiento conceptual de los estudiantes sobre la química de los ácidos, entre las cuales señalamos:

- Los ácidos tienen sabores amargos y olores penetrantes.

- Los ácidos son fuertes, poderosos y venenosos.

- Las sustancias que queman son ácidas.

- Toda sustancia que puede matar gérmenes es un ácido.

- Los ácidos no reaccionan con las bases; sólo se mezclan.

- Los ácidos deterioran las superficies sobre las que se encuentran.

- La fuerza de los ácidos causa "daño", "dolor" y "reactividad".

- Los ácidos en soluciones acuosas pueden permitir el paso de la corriente eléctrica.

El entendimiento sobre la química de los ácidos que revelan los estudiantes evidencia el predominio de las concepciones de tipo espontáneo (Carr, 1984), basadas en las experiencias y como producto de la percepción. Aunque estas concepciones son interpretaciones personales, se encontraron semejanzas entre lo expresado por los distintos estudiantes.

Pese a que los estudiantes que conformaron la muestra fueron divididos en tres grupos según los niveles académicos excelente, aceptable e insuficiente, al analizar los resultados se observa que no existen diferencias entre ellos, dado que manifiestan concepciones muy similares. 
Una característica importante en las concepciones espontáneas expresadas por los estudiantes consiste en que, al basar sus concepciones en las experiencias de tipo perceptivo, podían apreciar sólo las causas y efectos de los hechos observados sin establecer una distinción entre uno y otro, pasando por alto los fenómenos intermedios que ocurren en determinadas situaciones.

Las concepciones inducidas (Driver, 1988) que poseen los estudiantes son construcciones obtenidas a partir de la interacción con los medios de comunicación social, debido a que el estudiante tiene a su alcance una gran cantidad de información en donde la publicidad resulta ser una de las fuentes más influyentes (Arconada, 1994).

Las concepciones de los estudiantes se encuentran muy alejadas de las ideas aceptadas por la comunidad científica, considerándose muchas de ellas como errores conceptuales; tal afirmación se corrobora en los planteamientos de Vygotsky (1982), quien asume que los conceptos espontáneos y científicos se dan en direcciones opuestas: los primeros son manejados por el conocimiento que tiene el estudiante sobre determina-

\section{Bibliografía}

Arconada, M. A. (1994). Los consumidores de anuncios. Escola Crítica, 5:. 65-80.

Ausubel, D. P. Novack, J. D. y Hanesian, H. (1990). Psicología educativa, un punto de vista cognoscitivo. México: Editorial Trillas.

Barrios, A. M. (1997). Reflexiones epistemológicas y metodológicas en la enseñanza de las Ciencias para todos. (Tesis do concepto o fenómeno, mientras que los segundos son apropiaciones que se dan en el transcurso del trabajo en la escuela.

Las concepciones inducidas y analógicas (Pozo, et al., 1991) expresadas por los estudiantes sobre los ácidos guardan cierta relación, debido a que estas últimas surgen como interpretaciones personales pero muchas veces son construidas a partir de ideas relacionadas con el medio social. Es decir, las concepciones analógicas son producto de las concepciones inducidas que ya poseen los estudiantes.

Aparte de las concepciones de los estudiantes sobre la química de los ácidos como propósito del estudio, se evidenció la existencia de otras concepciones sobre temas de química que ya fueron abordados en el ámbito escolar (Furio y otros, 2000; Zafra, 2001 y Pérez, 1991), lo cual permite establecer que existe la persistencia de estas ideas a pesar de la enseñanza impartida. Todo esto se debe, según Cazden (1989), a que las concepciones alternativas son difíciles de desaparecer porque parecen naturales para quien aprende y son adquiridas en el transcurso de un período largo. $\boldsymbol{\Delta}$

de Maestría) Montevideo: Universidad de Montevideo.

Borsese, A. (1992). "Fuerzas de los ácidos y de las bases y criteri os de cálculo de $\mathrm{pH}$ ”. Enseñanza de las Ciencias, 10 (1), 86-88.

Carr, M. (1984). "Model confusion in Chemistry", Research in Science Education, 14:97-103. 
Cazden (1989 y 1991). Citado en página de Internet. www.pue.uía.mx/docedu/ revista/revistaDOS/tesis.html

Driver, R. (1988). "Un enfoque constructivista para el desarrollo del currículo en Ciencia”. En: Osborne, R. y Freyberg, P. (eds), El aprendizaje de las Ciencias: influencia de las ideas previas de los alumnos. Madrid, España: Editorial Narcea.

Driver y Gasley, 1978

Fensham, P., Gunstone, R. y White, R. (eds.), (1994) The Falmer Press.

Furio, C., Calatayud, M. L. y Bárcenas, S. (2000). "Deficiencias epistemológicas en la enseñanza de las reacciones ácido-base y dificultades de aprendizaje”. En: Revista TED, N. ${ }^{\circ}$ 7. Santafé de Bogotá: Facultad de Ciencias y Tecnología, Universidad Pedagógica Nacional. Furio y otros, 2000

Gilbert, 1982

Giordan, 1985

Giordan, A. y De Vecchi, G. (1988). Los orígenes del saber. Sevilla: Díada.

Hand, B. (1989). "Student understanding of acids and bases: a to year study", Research in Science Education, 19:133-144.

Hand y Treagust (1989)

Hewson, P. y A Beckett Hewson, M. (1984). "The role of conceptual conflict in conceptual change and design of Science instruction". Instructional Science, 13 (1), 1-13.

Maykut, P. y Morehouse, R. (1994). Beginning qualitative research. A philosophic and practical guide. Londres: The Falmer Press.

Nakhleh, M. (1994). "How can research uncover what students are learning?" Journal of Chemical Education, 71(3), 201-203.
Osborne, R. y Wittrock, M. (1983). "Learning Science: a generative process". Science Education, 67: 489-508.

Pérez, R. (1999). “Aprendizaje de los conceptos de ácido y de base en estudiantes de educación media Vocacional: Una estrategia pedagógica fundamentada en la teoría de Novak sobre la educación". Tesis inédita. Santafé de Bogotá: Facultad de Ciencias y Tecnología, Universidad Pedagógica Nacional

Platten, M. (1991). “Teaching concepts and skills of thinking simultaneously". (ERIC Document Reproduction Service $\mathrm{N}^{\circ}$ ED 338543).

Pozo, J. I., Sanz, A., Gómez Crespo, M. A. y Limón, M. (1991). "Las ideas de los alumnos sobre la ciencia: una interpretación desde la psicología cognitiva”. Enseñanza de las Ciencias, 9: 83-94.

Solomon, J. (1987). "Social influences on the construction of pupils' understanding of Science". Studies in Science Education, 14: 63-82.

Taramazza, 1981

Trilla, J. (1993). La educación en la escuela. Barcelona: Arial.

Vygotsky, L. S. (1982). Obras escogidas II. Problemas de psicología general. Madrid: Visor Distribuidores.

Zafra, S. (2001).

Zafra, S. (2001). "El aprendizaje total de los conceptos científicos ácidobase". Tecné, Episteme y Didaxis, № 10. 66-78. 